\title{
Tumor rectal como presentación de sífilis primaria*
}

\author{
Drs. GINO CASELLI M. ${ }^{1}$, GEORGE PINEDO M. ${ }^{1}$, AL. SERGIO NIKLITSCHEK L. ${ }^{2}$ \\ Departamento de Cirugía Digestiva, Hospital Clínico, Pontificia Universidad Católica de Chile. \\ 2 Escuela de Medicina. Pontificia Universidad Católica de Chile, Santiago, Chile.
}

\begin{abstract}
Rectal tumor as a presentation of primary syphilis

In the era of HIV infection there is a resurgence of syphilis. Without a doubt it is still a diagnostic dilemma in various clinical situations such as anorectal syphilis in HIV patients. When a patient HIVpositive presents with a rectal tumor, syphilis must be considered in the differential diagnosis. We present a case report of an HIV positive patient who consulted due to rectal tenesmus associated to rectal bleeding, being diagnosed a rectal tumor and treated secondary to primary syphilis.
\end{abstract}

Key words: HIV infection, Treponema Pallidum, rectal syphilis.

\section{Resumen}

En la era de la infección por VIH, ha habido un resurgimiento de enfermedades en franca disminución en el mundo occidental como sífilis. Sin lugar a dudas siguen siendo un dilema diagnóstico algunas manifestaciones clínicas como sífilis rectal en pacientes portadores de VIH. Frente a un tumor rectal en un paciente VIH se debe tener en cuenta en el diagnóstico diferencial a esta patología. Presentamos un caso de un paciente portador de VIH que consultó por tenesmo rectal asociado a rectorragia, siendo diagnosticado un tumor rectal secundario a sífilis primaria.

Palabras clave: Infección por VIH, Treponema Pallidum, sífilis rectal.

\section{Introducción}

Existe clara evidencia en la literatura, en los últimos años, del creciente aumento en la incidencia de sífilis en el mundo. Esta enfermedad sistémica y de transmisión sexual afecta de forma mayoritaria a un grupo etario que comprende la tercera década de la vida. Los hombres homosexuales representan el grupo de más alto riesgo. La sífilis anorrectal es una rara manifestación de esta enfermedad, que pudiera ser asintomática, y sus lesiones pueden pasar fácilmente inadvertidas ${ }^{1}$ como proctitis, ulceraciones o pseudotumores. Por lo tanto, los cirujanos que realizan procedimientos endoscópicos deben estar alertos para evitar diagnósticos incorrectos, tratamientos inapropiados y no retrasar el manejo antibiótico.

\section{Caso Clínico}

Se presenta el caso de un paciente de sexo masculino de 37 años de edad, portador del virus de

\footnotetext{
* Recibido el 6 de Octubre de 2008 y aceptado para publicación el 9 de Diciembre de 2008.

Correspondencia: Dr. George Pinedo Mancilla

Marcoleta 367, Santiago, Chile

E-mail: gpinedo@med.puc.cl
} 


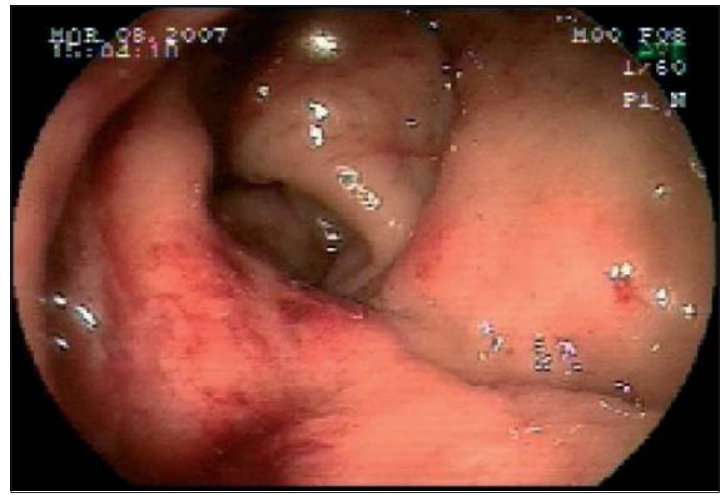

Figura 1. Colonoscopia.

inmunodeficiencia humana diagnosticado en Noviembre de 1999, que en Febrero del año 2007 consulta por dolor anal de meses de evolución y que se acentúa al defecar. A la inspección se identifica una fisura anal crónica en la comisura posterior. $\mathrm{Al}$ tacto rectal se palpa una masa circunferencial ubicado sobre el anillo anorrectal.

Se realiza además una rectoscopia rígida que explora hasta $25 \mathrm{~cm}$ del margen anal, identificando en el límite entre el canal anal y recto inferior una lesión ulcerada, solevantada, blanquecina de aspecto neoplásico. El informe de la biopsia señala la existencia de inflamación crónica leve y compromiso extenso por neoplasia intraepitelial grado 2 del epitelio escamoso anal, sin evidencias de infiltración. Se continúa el estudio con colonoscopia total que observa a nivel del canal anal y ampolla rectal una lesión solevantada, ulcerada que sangra con facilidad (Figura 1). El examen microscópico concluye colitis crónica activa, erosiva, moderadamente intensa y granulomatosa. La resonancia magnética pelviana informa extenso compromiso tumoral infiltrativo transmural de los dos tercios inferiores del recto, alcanzando hasta el margen superior del canal anal (Figura 2).

Además existe importante infiltración intersticial del mesorrecto. Hay adenopatías en el mesorrecto de $1,9 \mathrm{~cm}$ y extensas adenopatías ilíacas externas bilaterales. Se decide realizar biopsia de tumor rectal con aguja de trucut en masa perirrectal, cuyo informe señala la observación de espiroquetas en microscopio electrónico de campo oscuro. Además se realizó test RPR treponémicos (MHA-TP y ELISA Captia Syphilis-G y Pathozyme Syphilis-M) cuya reacción se informó reactivo en dilución 1:32 en Abril del 2007 y reactivo 1:8 en Mayo del 2007 (se contaba con un test serológico negativo en Noviembre del 2006). Se decidió iniciar tratamiento antibiótico con penicilina benzatina 2,4 millones de

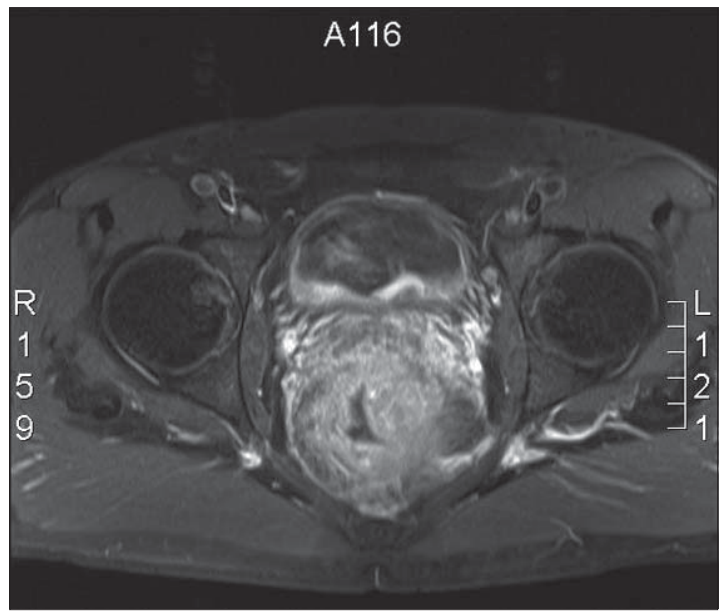

Figura 2. RNM pelviana.

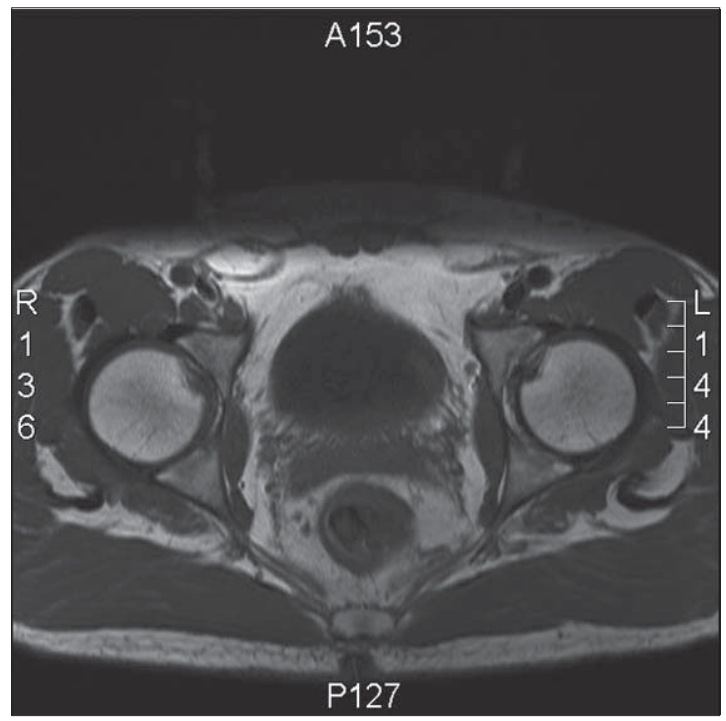

Figura 3. RNM pelviana de control.

unidades por vía intramuscular, 2 veces por 2 semanas consecutivas. Tres meses de culminado el tratamiento se le realiza nueva RNM de pelvis de control que muestra mínimo engrosamiento residual de la pared del recto distal, no observando adenopatías en el tejido adiposo mesorrectal (Figura 3).

\section{Discusión}

La sífilis anorrectal es un diagnóstico poco habitual al enfrentar una masa rectal; sin embargo, en 
el grupo de pacientes HIV positivo este diagnóstico debe estar en el armamentario del clínico. El centro de control y prevención de enfermedades de US señala que existe un preocupante aumento de casos de sífilis en las naciones desarrolladas. De hecho, el número de casos se ha incrementado 12 veces en los últimos cinco años sólo en el Reino Unido $^{2}$. La característica más constante de la sífilis en el canal anal es la ulceración ${ }^{3}$. La evaluación temprana de los síntomas y signos en este caso eran muy sugerentes de una neoplasia rectal, sin embargo, los estudios histológicos nunca mostraron etiología tumoral. La ubicación en la pared anterior es típica de la sífilis primaria anorrectal, como es reportado en otros $\operatorname{casos}^{4,5}$.

La sífilis anorrectal puede manifestarse de distintas formas que varían de caso a caso, siendo a menudo asintomáticas. La presentación a nivel anorrectal en sífilis es habitualmente en relación a lesiones anales (fisuras, úlceras y condilomas acuminados $)^{6}$. En general las lesiones rectales, que se presentan como proctitis, pólipos, úlceras o pseudotumores son menos frecuentes. El diagnóstico diferencial debe incluir enfermedades inflamatorias intestinales ${ }^{7}$ (en especial la enfermedad de Crohn), proctitis de otra etiología, pólipos neoplásicos o benignos, úlcera rectal solitaria, linfomas, linfogranulomas venéreos, tuberculosis ${ }^{8}$ y cáncer rectal (adenocarcinoma o carcinoma indiferenciado). Tal como señalan distintos autores, la serología pudiera ser negativa aún cuando la lesión primaria esté desarrollada, siendo necesaria la repetición de estos estudios, ya que un estudio negativo no excluye una etiología sifilítica. Sin embargo, estas pruebas (las de laboratorio para la enfermedad venérea -VDRL-y la prueba rápida con reagina plasmática -RPR) tienen baja especificidad y sensibilidad en los casos de sífilis primaria y en las etapas tardías de la infección. Por su parte las pruebas treponémicas - como la que detecta el Treponema Pallidum mediante la aglutinación de partículas -(TP-PA) están dirigidas a anticuerpos específicos contra esta espiroqueta y son más sensibles debido a que los anticuerpos contra el Treponema preceden por lo general a los anticuerpos antilipídicos. En general, la sensibilidad de la prueba TP-PA es mayor que la de VDRL y la de RPR ( $88 \%$ frente a $78 \%$ y $86 \%$ respectivamente) ${ }^{9}$. Aún teniendo en cuenta estas pruebas, el diagnóstico de referencia según muchos autores es el examen por microscopia de campo oscuro $(\mathrm{MCO})^{10}$.

Cabe destacar que la neoplasia intraepitelial del canal anal no es infrecuente en pacientes VIH positivos infectados con Virus papiloma humano ${ }^{11}$, por lo cual no sorprende que este paciente presente esta neoplasia del canal anal en una biopsia de teji- do del canal, aunque estas alteraciones no fueron el verdadero motivo de consulta.

El tratamiento es realizado con antibióticos habitualmente penicilina benzatina. En nuestro caso después de tres meses el paciente presentó una mejoría sintomática y se evidenció una clara disminución del tamaño de la masa tumoral.

\section{Conclusiones}

Creemos que médicos que tratan enfermedades colorrectales deben mantenerse en alerta ante el aumento en la incidencia de sífilis en las últimas décadas. De hecho, la falla en la realización del diagnóstico de sífilis del anorrecto descansa en dos factores: En primer lugar, los clínicos no reconocen la variedad de manifestaciones de la sífilis primaria y segundo, el diagnóstico inadecuado es a consecuencia de la falla en mantener una alta sospecha al manejar lesiones en la región anorrectal en pacientes con factores de riesgo ${ }^{12}$. Cualquier lesión atípica en pacientes VIH positivo con patología anorrectal benigna debe alertar sobre la posibilidad de sífilis.

\section{Referencias}

1. Quinn T, Lukehart S, Goodell S, Mkrtichian E, Schuffler M. Rectal mass caused by Treponema Pallidum: confirmation by inmunofluorecent stain. Gastroenterology 1982; 82: 135-139.

2. Fenton K, Breban R, Vardavas R, Okano J, Martin T. Infectious syphilis in high-income settings in the $21 \mathrm{st}$ century. The Lancet Infectious Diseases 2008; 8: 224253.

3. Morson B. Anorectal venereal disease. Proc R Soc Med 1964; 57: 179.

4. Gluckmann J, Kleinmann M, May A. Primary syphilis of rectum. NY State J Med 1974; 74: 2210-2211.

5. Wells B, Kierland R, Jackmann R. Rectal cancer: report of a case. Arch Dermatol 1959; 79: 719-721.

6. Bassi O, Cosa G, Colavolpe A, Argentieri R. Primary syphilis of the rectum-Endoscopic and clinical features. Report of a case. Dis Colon Rectum 1991; 34: 10241026.

7. Le Marchant J, Ferrand B. Rectite syphilitique primarie associee a une atteinte hepatique. Sem Hop Paris 1981; 57: $1434-1438$

8. Leonidas G, Koniaris, Jeffrey L, Seibel. Tuberculosis presenting as a perirectal mass. Dis Col Rectum 2000; 43: $1604-1605$.

9. Nueva estrategia de diagnóstico para la sífilis primaria. Revista Panamericana de Salud Pública 2008; 23. Disponible en: http://www.scielo.org/ (consultado el 03 de Julio del 2008). 
10. Drusin L, Homan W, Dineen P. The role of surgery in primary syphilis of the anus. Ann Surg 1976; 184: 65-67.

11. Berry J, Palefsky J, Welton M. Anal cancer and its precursors in HIV-positive patients: Perspectives and management. Surg. Oncol Clin N Am 2004; 13: $355-$ 373.

12. Sung S, Jang I, Kim B, Kim E, Woo S. A case of primary syphilis in the rectum. J Corean Med Sci 2005; 20: $886-887$. 